\title{
Propiedades biomecánicas de la membrana limitante interna tras recibir tratamiento intravítreo con ocriplasmina
}

\author{
Franziska Vielmuth $^{\mathrm{a}}$ Ricarda G. Schumann ${ }^{\mathrm{b}}$ Volker Spindler ${ }^{\mathrm{a}}$ Armin Wolf $^{\mathrm{b}}$ \\ Renate Scheler $^{b}$ Wolfgang J. Mayer ${ }^{b}$ Paul B. Henrich ${ }^{d}$ Christos Haritoglou $^{b, c}$ \\ a Departamento I, Instituto de Anatomía y Biología Celular, y bepartamento de Oftalmología, Universidad de \\ Múnich, y ${ }^{\mathrm{C} H e r z o g}$ Carl Theodor Eye Hospital, Múnich, Alemania; ${ }^{\mathrm{d} D e p a r t a m e n t o ~ d e ~ O f t a l m o l o g i ́ a, ~ K a n t o n s s p i t a l ~}$ \\ Winterthur, Winterthur, Suiza
}

\section{Palabras clave}

Membrana limitante interna · Ocriplasmina · Tracción

vitreomacular

\section{Resumen}

Objetivo: Evaluar la rigidez de la membrana limitante interna (MLI) humana y evaluar los posibles cambios de las propiedades mecánicas tras administrar una inyección intravítrea de ocriplasmina para tratar la tracción vitreomacular. Métodos: Este estudio se compone de una serie de casos intervencionales y comparativos de 12 muestras de MLI extraídas mediante cirugía y obtenidas de forma consecutiva de 9 ojos de 9 pacientes después de someterse sin éxito a vitreólisis farmacológica con ocriplasmina. Durante el mismo periodo de tiempo, 16 muestras de otros 13 ojos sin tratamiento con ocriplasmina se obtuvieron mediante vitrectomía y sirvieron como controles. Todos los pacientes presentaron agujeros maculares o tracción vitreomacular y se sometieron a vitrectomía con disección de la MLI tanto con tinción con azul brillante $(A B)$ como sin ella. Todas las muestras se analizaron con un microscopio de fuerza atómica con imágenes de las regiones de $25 \times 25 \mu \mathrm{m}$. En todas las muestras, se analizaron tanto la parte de la retina como la del vítreo de la MLI. Resultados: La microscopia de fuerza atómica no reveló diferencias significativas en cuanto a elasticidad

\section{KARGER}

(C) 2017 S. Karger AG, Basel

E-Mail karger@karger.com

www.karger.com/oph de las muestras de MLI extraídas de ojos con o sin tratamiento con ocriplasmina. Las áreas onduladas de la parte de la retina presentaron una mayor rigidez que la parte del vítreo de la MLI. La cartografía topográfica tanto de la parte del vítreo como de la retina de la MLI no mostró ninguna alteración aparente de la morfología en ojos tratados con ocriplasmina en comparación con los ojos no tratados. La tinción con azul brillante conllevó un aumento de la rigidez tisular. Conclusiones: Las inyecciones intravítreas de ocriplasmina no varían las propiedades biomecánicas de la MLI humana. No existen pruebas de un posible efecto enzimático que interfiera con la rigidez de esta membrana basal.

(c) 2017 S. Karger AG, Basel

\section{Introducción}

La ocriplasmina (Jetrea ${ }^{\circledR}$, ThromboGenics, Lovaina, Bélgica) constituye una forma truncada sin especificar y recombinante de la serina proteasa plasmina que posee actividad proteolítica frente a la laminina y fibronectina, ambos componentes principales de la interfase vitreore-

The Spanish version should be cited as DOI: 10.1159/000477510. The original version is in English and should be cited as DOI: 10.1159/000444508. 
tiniana [1]. Recientemente se aprobó la vitreólisis farmacológica con ocriplasmina para el tratamiento de pacientes con adhesión vitreomacular (AVM) y tracción vitreomacular (TVM) sintomática, también cuando estuviera asociada a agujeros maculares de grosor completo de $\leq 400 \mu \mathrm{m}$ de diámetro $[2,3]$. La liberación de la TVM puede conseguirse mediante vitreólisis farmacológica hasta en el $70 \%$ de los casos tras administrar una única inyección intravítrea de ocriplasmina en la cavidad vítrea $(0,125 \mathrm{mg} / 0,1 \mathrm{ml})$, tal y como se confirmó en análisis de subgrupos de ojos con TVM $[4,5]$.

En comparación con la vitrectomía a través de la parte plana con disección de la membrana limitante interna (MLI), que hasta hace muy poco comprendía la única opción de tratamiento, la inyección intravítrea de ocriplasmina supone una intervención terapéutica generalmente bien tolerada en pacientes con TVM $[6,7]$. La vitreólisis farmacológica consta de numerosas posibles ventajas sobre la vitrectomía quirúrgica, tales como la posibilidad de evitar riesgos quirúrgicos, una rehabilitación visual más rápida y la inducción de un desprendimiento de vítreo posterior completo sin restos de colágeno en la corteza vítrea de la MLI.

No obstante, surgieron ciertas inquietudes sobre el perfil de seguridad de la ocriplasmina. Un análisis más detallado de datos de seguridad procedentes de ensayos en fase II y III, así como casos clínicos y pequeñas series de casos revelaron anomalías estructurales y funcionales panretinianas agudas después del tratamiento con ocriplasmina que no parecen estar relacionadas tan solo con la inducción de un desprendimiento de vítreo posterior completo. Se podría suponer que, a la luz de tal información, la ocriplasmina afecta de algún modo a estructuras o componentes celulares más profundos de la retina, importantes para la integridad retiniana. En este caso, la ocriplasmina tendría que atravesar la MLI. Teniendo en cuenta las propiedades enzimáticas de la ocriplasmina, se podría asumir que la ocriplasmina también afecta a los componentes conocidos de la MLI, tales como la laminima y la fibronectina. Este efecto probablemente afectaría a las propiedades biomecánicas de la MLI, que constituye un tejido muy rígido, y cuya rigidez biomecánica se asemeja al cartílago articular.

La microscopia de fuerza atómica (MFA) representa una técnica sólida que mide la rigidez tisular de las membranas basales, tales como la MLI a un nivel de nanoescala [8-11]. En el presente estudio experimental, utilizamos esta técnica para cuantificar los cambios potenciales de las propiedades biomecánicas de la MLI tras el tratamiento con ocriplasmina para maculopatías traccionales, tales como agujeros maculares o síndrome de TVM.

\section{Materiales y métodos}

Este estudio se compone de una serie de casos intervencionales y comparativos de 12 muestras de MLI extraídas mediante cirugía y obtenidas de forma consecutiva de 9 ojos de 9 pacientes después de someterse sin éxito a vitreólisis farmacológica con ocriplasmina. Los pacientes se sometieron a vitrectomía con disección de la MLI en el Departamento de Oftalmología de la Universidad Ludwig Maximilian, y en el Herzog Carl Theodor Eye Hospital, de Múnich, entre mayo de 2014 y enero de 2015. El Comité Institucional de Revisión y el Comité Ético de la Universidad Ludwig Maximilian de Múnich aprobaron la revisión retrospectiva de los datos de los pacientes, así como la preparación y el análisis de las muestras ( $\mathrm{N}^{\circ}$ 471-14). Este estudio se llevó a cabo de acuerdo con los principios de la Declaración de Helsinki.

6 ojos mostraron agujeros maculares persistentes y 3 presentaron TVM sintomática persistente, según confirmó la tomografía de coherencia óptica de alta resolución. 5 de los 6 ojos con agujeros maculares se observaron con resolución de TVM y progresión del diámetro del agujero macular tras inyección de ocriplasmina. Se realizó cirugía macular tras un periodo medio de 4,1 meses (intervalo 1-10 meses) posterior a la inyección de ocriplasmina. Los ojos con agujeros maculares se sometieron a cirugía tras un periodo medio de 2,3 meses (intervalo 1-3 meses), que fue significativamente más temprano que en el caso de los ojos con TVM. Se aplicó vitreólisis farmacológica con ocriplasmina de acuerdo con las directrices de la Sociedad Oftalmológica Alemana, la Sociedad de Retina y la Asociación Profesional de Oftalmólogos en Alemania sobre la administración intravítrea terapéutica de ocriplasmina.

Durante el mismo periodo de tiempo, se obtuvieron mediante vitrectomía 16 muestras de otros 13 ojos sin tratamiento con ocriplasmina y sirvieron como controles. En este grupo de control, incluimos 4 muestras de 4 ojos con agujeros maculares idiopáticos y 12 muestras de 9 ojos con TVM.

En 3 ojos con tratamiento con ocriplasmina, se extrajeron 2 muestras durante la vitrectomía, respectivamente. En estos casos, la primera muestra se extrajo sin tinción intravítrea de la MLI durante la cirugía; la segunda muestra se extrajo con el uso del tinte vital azul brillante $(\mathrm{AB})$. De manera similar, en 3 ojos del grupo de control sin tratamiento con ocriplasmin, se extrajeron 2 muestras por ojo durante la vitrectomía, tal y como se comenta más arriba.

\section{Procedimiento quirúrgico y extracción de muestras}

La técnica quirúrgica consistió en una vitrectomía a través de la parte plana $23 \mathrm{G}$ a tres puertos con disección de la MLI. Todos los pacientes fueron operados por 3 cirujanos experimentados. La vitrectomía se realizó de la siguiente manera: antes de abrir la línea de infusión, el estado del hialoide posterior se determinó con una lente de contacto plano-cóncava. Si el vítreo estaba sujeto o parcialmente sujeto a la retina, se inducía el desprendimiento de vítreo posterior mediante succión con la sonda de vitrectomía sobre el disco óptico o en la mitad de la periferia. El hialoide posterior se desprendía de la retina y se extraía hacia la periferia.

Para mejorar la precisión de la disección de la MLI y para evitar una extracción incompleta, se utilizó tinción con azul brillante $G$ (ABG; 0,02 ml, Brilliant Peel; Geuder, Heidelberg, Alemania) para visualizar la MLI en 7 ojos con tratamiento con ocriplasmina previo y en 8 ojos sin tratamiento con ocriplasmina. El resto de los ojos se operaron sin tinción intraoperativa de la MLI. En un subgrupo de ojos con tinción $\mathrm{AB}$, la primera muestra de MLI se extra-
2

Ophthalmologica 2017;238(suppl 1):1-8 DOI: $10.1159 / 000477510$
Vielmuth/Schumann/Spindler/Wolf/ Scheler/Mayer/Henrich/Haritoglou 
jo sin $\mathrm{ABG}$, y la segunda muestra se extrajo con $\mathrm{AB}$. En el resto de los ojos, tan solo se extrajo una muestra. En caso de que se aplicara, la solución de tinte se inyectó en la interfase vitreomacular e inmediatamente se lavó. No se aplicó ningún otro tinte. La disección de la MLI se realizó con pinzas de Eckardt. En caso de que fuera necesario, la extracción de la MLI se realizó en bloque con tejido epirretiniano.

La cirugía se completó revisando si había roturas en la periferia de la retina. En ojos con pequeños agujeros maculares, la cavidad vítrea se perfundió con una mezcla del $15 \%$ C2F6 tras un intercambio de fluido/aire. En ojos con adhesión vitreomacular persistente, se dejó una solución salina equilibrada en la cavidad vítrea al final del procedimiento. Las muestras de MLI obtenidas se extrajeron e inmediatamente se colocaron sobre portaobjetos de vidrio sin fijación.

\section{Microscopia de fuerza atómica (MFA)}

En el análisis con microscopio de fuerza atómica, se utilizó un NanoWizard ${ }^{\circledR} 3$ AFM (JPK-Instruments, Berlín, Alemania) montado en un microscopio invertido (Carl Zeiss, Jena, Alemania), lo que permite seleccionar el área de escáner exacta visualizando la MLI con un objetivo de $10 \times$ (fig. 1a). Las muestras se montaron en los portaobjetos del microscopio y se sometieron a las mediciones de la MFA para delinear la topografía y elasticidad. En cada muestra se seleccionó la medición a partir de la imagen óptica en la que se veía claramente la membrana vacía sin células epirretinianas o colágeno (fig. 1a). La morfología habitual de la parte retiniana rugosa y de la parte vítrea más bien lisa también se delineó mediante imágenes de altura del MFA y tinción de la laminina (fig. 1b, c). A lo largo de todos los experimentos, la MFA operó en modo QI, un modo de técnica de imagen basado en espectroscopia de fuerza que permite realizar mediciones simultáneas de la topografía y elasticidad de las muestras.

Se utilizaron cantilévers de $\mathrm{Si}_{3} \mathrm{~N}_{4}$ con puntas $\mathrm{D}$ en forma de pirámide (MLCT, Bruker, Mannheim, Alemania) con una constante de resorte nominal de $0,03 \mathrm{~N} / \mathrm{n}$. La constante de resorte $\mathrm{k}$ se determinó con el método de ruido termal [12]. Las muestras se midieron bajo condiciones fisiológicas a $37^{\circ} \mathrm{C}$ en PBS. En cuanto a los cuadros sinópticos, se eligieron áreas de barrido amplias de $25 \times$ $50 \mu \mathrm{m}$ con una resolución de $512 \times 256$ píxeles (fig. $1 \mathrm{a},{ }^{*}$ ). Para los mapas de elasticidad, se eligieron áreas de barrido amplias de $25 \times$ $25 \mu \mathrm{m}$ (3 áreas/lado distintas) a partir de la imagen óptima y la resolución se estableció a $64 \times 64$ píxeles (fig. 1a,\#). Para cada píxel, se registró información de la altura (para generar mapas topográficos) y elasticidad (para calcular el módulo de Young, MY). En total, se obtuvieron 3 áreas con 4.096 mediciones cada una para cada muestra. Se aplicó una longitud z de $2.000 \mathrm{~nm}$, una velocidad de extensión y retroceso de $100 \mu \mathrm{m} / \mathrm{s}$ (mapas panorámicos) o 10 $\mu \mathrm{m} / \mathrm{s}$ (mapas de elasticidad), un retroceso adicional de $200 \mathrm{~nm}$ y un punto fijo relativo de $0,2 \mathrm{nN}$ para evitar alteraciones de muestra debido a la tensión mecánica. Para la determinación del MY, se aplicó el programa de análisis JPK, que calcula el MY con la fórmula Bilodeau para el indentador piramidal del modelo de Hertz $[13,14]$.

Debido a la delineación simultánea de topografía y elasticidad, se pudo especificar durante las mediciones la orientación de la muestra con diferenciación entre la parte vítrea y la retiniana de la MLI. Se llevaron a cabo pruebas adicionales tras las mediciones de la MFA con el objetivo de confirmar la orientación de las muestras. Todas las muestras se fijaron con una solución de paraformalde-

Propriedades de la MLI tras recibir tratamiento intravítreo con ocriplasmina hído 2 \% y se procesaron para su inmunotinción indirecta con anticuerpos de ratón antilaminina humana (clon 4C7; Dako, Hamburgo, Alemania) y un anticuerpo secundario Cy3 capruno-murino. La captura de las imágenes se realizó con un microscopio confocal SP5 equipado con objetivos Plan Apo 20× NA 0,7 y Plan Apo $63 \times$ NA 1,4 (ambos Leica, Mannheim, Alemania; fig. 1c).

\section{Análisis de datos y estadísticas}

Todas las muestras extraídas por cirugía se procesaron con enmascaramiento y fueron analizadas por 2 investigadores de forma independiente según 4 grupos de la siguiente manera: (1) muestras tras tratamiento con ocriplasmina extraídas con uso intravítreo de $\mathrm{AB} ;(2)$ muestras tras tratamiento con ocriplasmina extraídas sin tinción intravítrea de la MLI; (3) muestras sin tratamiento con ocriplasmina extraídas con uso intravítreo de $\mathrm{AB}$ y (4) muestras sin tratamiento con ocriplasmina extraídas sin tinción intravítrea de la MLI.

Las imágenes y el MY se procesaron con el programa de procesamiento de datos JPK. Se llevó a cabo un análisis adicional con Adobe Photoshop CS5. Los valores de datos se compararon con un análisis de la varianza unidireccional con pruebas de comparación múltiple de Kruskal-Wallis y Tukey en Prism (Graphpad Software, La Jolla, Calif., EE. UU.). Se supuso una significación estadística de $\mathrm{p}<0,05$. Los datos se muestran como media \pm error estándar de la media.

\section{Resultados}

La microscopia de fuerza atómica no reveló diferencias significativas en cuanto a elasticidad de las muestras de MLI extraídas de ojos con o sin tratamiento con ocriplasmina. Las muestras extraídas de ojos tratados con ocriplasmina pero sin tinción con BB indicaron un ligero aunque no signficativo aumento del MY de 34,18 \pm 8,47 $\mathrm{kPa}$ en la parte vítrea y de $148,11 \pm 27,29 \mathrm{kPa}$ en la parte retiniana de la MLI. En cambio, en los ojos no tratados del grupo de control, la MLI demostró un MY de 16,29 \pm $3,34 \mathrm{kPa}$ en la parte vítrea y de $122,76 \pm 26,64 \mathrm{kPa}$ en la parte retiniana (fig. 2a, MY). De acuerdo con las mediciones de topografía y elasticidad, las áreas onduladas de la parte retiniana se presentaron más rígidas (fig. 2a, MY, flechas blancas). La cartografía topográfica tanto de la parte del vítreo como de la retina de la MLI no mostró ninguna alteración aparente de la morfología en ojos tratados con ocriplasmina en comparación con ojos sin inyección de ocriplasmina previa vitrectomía (fig. 2a). De manera similar, no se encontraron alteraciones en la topografía al comparar la disección de la MLI no teñida y la disección de la MLI con AB.

Al comparar muestras de la MLI extraídas con y sin administración intravítrea de $\mathrm{AB}$, se observó una diferencia en el MY entre las muestras extraídas con y sin $\mathrm{AB}$. $\mathrm{La}$ administración intravítrea de $\mathrm{AB}$ para conseguir una

Ophthalmologica 2017;238(suppl 1):1-8 DOI: $10.1159 / 000477510$ 


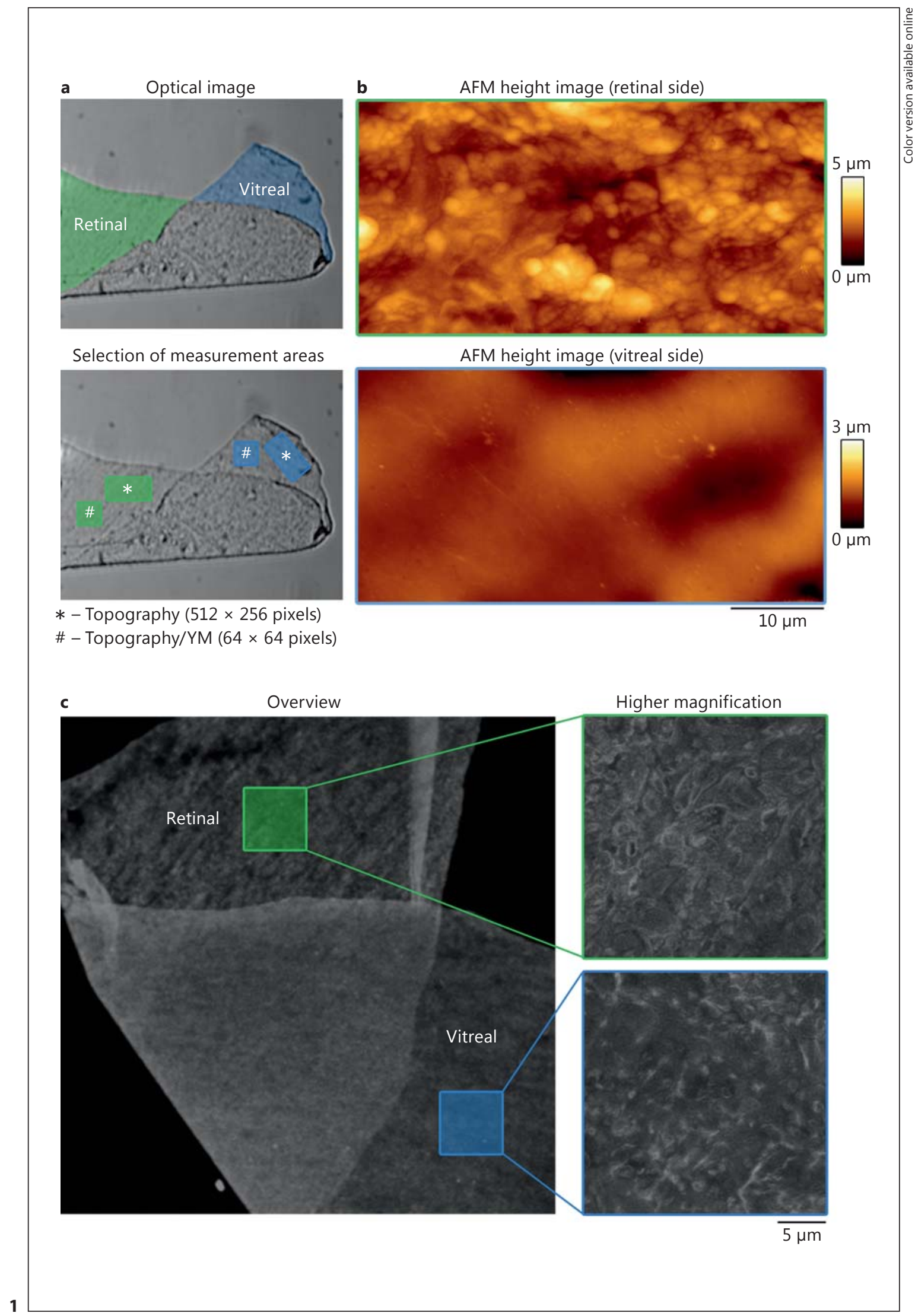

(For legend see next page.) 
disección de la MLI sencilla y segura conllevó un MY superior, que se mostró especialmente notable en la parte vítrea (fig. 2b). En los ojos no tratados con ocriplasmina, el uso de AB implicó un aumento significativo del MY de $16,29 \pm 3,34 \mathrm{kPa}$ en la parte vítrea y de $122,76 \pm 26,64 \mathrm{kPa}$ en la parte retiniana. En ojos no teñidos, el MY dio como resultado $68,17 \pm 18,74 \mathrm{kPa}$ en la parte vítrea y $152,1 \pm$ $17,46 \mathrm{kPa}$ en la parte retiniana de la MLI. En ojos tratados con ocriplasmina, el aumento tras la tinción con BB fue menos pronunciado con un MY de $34,18 \pm 8,47 \mathrm{kPa}$ en la parte vítrea y de $148,11 \pm 27,29 \mathrm{kPa}$ en la parte retiniana de la MLI no teñida, en comparación con un MY de
$49,42 \pm 10,89 \mathrm{kPa}$ en la parte vítrea y de $166,65 \pm 31,49$ $\mathrm{kPa}$ en la parte retiniana de la MLI en ojos con disección de la MLI con AB (fig. 2a, b).

Para estudiar el efecto de la disección de la MLI con $\mathrm{AB}$ en ojos con ocriplasmina, investigamos muestras de 3 ojos sometidos a vitrectomía con disección de una primera muestra de MLI no teñida seguida de la administración de $\mathrm{AB}$ con disección de una segunda muestra de MLI teñida. Se llevó a cabo un análisis de topografía y elasticidad en ambas muestras, tal y como se comenta más arriba. El MY antes de la tinción se estableció en 1,0 (fig. 3a). Los mapas topográficos confirman que la mor-

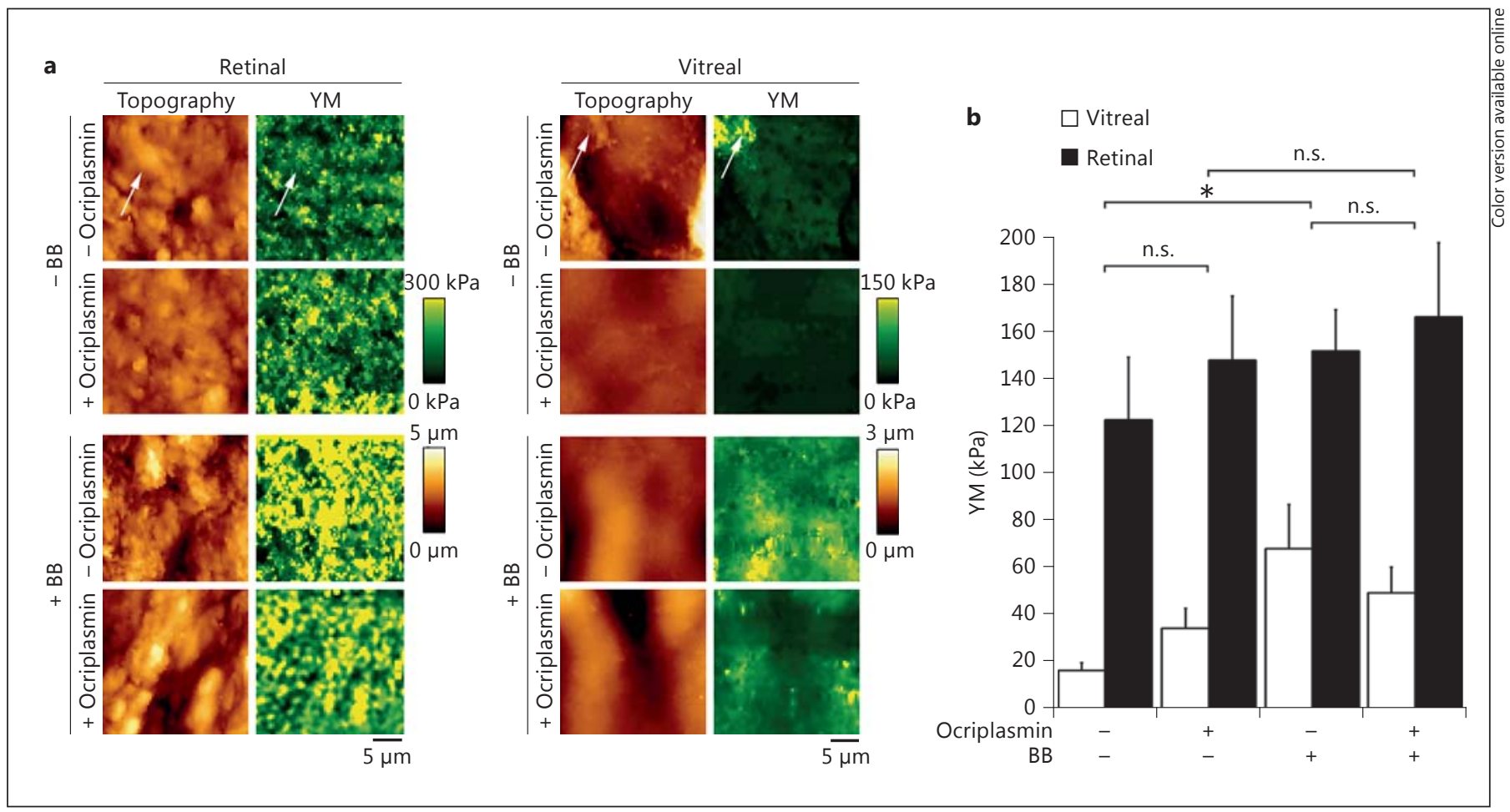

Fig. 2. a Los mapas topográficos y de elasticidad de la parte de la retina (cuadros izquierdos) y del vítreo (cuadros derechos) de la MLI no revelan cambios morfológicos o de elasticidad debido al tratamiento con ocriplasmina. La topografía y el MY se encuentran relacionados en varias áreas (flechas blancas). El AB aumenta el
MY tanto en el grupo de control como en el de ocriplasmina. b Promedio de todos los mapas en los respectivos grupos para ambos lados de la MLI con barras de error que representan el error estándar de la media. ${ }^{*} \mathrm{p}<0,05$.
Fig. 1. a Imagen óptica de MLI disecada. La MLI está parcialmente volteada para exponer la parte vítrea y retiniana. La parte de la retina aparece marcada en verde; la parte del vítreo aparece marcado en azul (imagen superior). Las áreas de barrido (rectángulos verdes/azules) fueron seleccionadas en primera instancia para identificar la parte respectiva de la MLI $(50 \times 25 \mu \mathrm{m}, 512 \times 256$ píxeles, $\left.{ }^{*}\right)$ y, después, para medir la topografía y elasticidad en ambos lados $(25 \times 25 \mu \mathrm{m}, 64 \times 64$ píxeles, \#). b Parte retiniana rugosa y ondulada y parte vítrea lisa de la MLI. c MLI teñida para visualizar la laminina por microscopio de fluorescencia. De nuevo, las partes de la retina, rugosa, y del vítreo, lisa, se distinguen claramente. Para ver los colores, observe la versión en línea.
Propriedades de la MLI tras recibir tratamiento intravítreo con ocriplasmina
Ophthalmologica 2017;238(suppl 1):1-8 DOI: $10.1159 / 000477510$ 


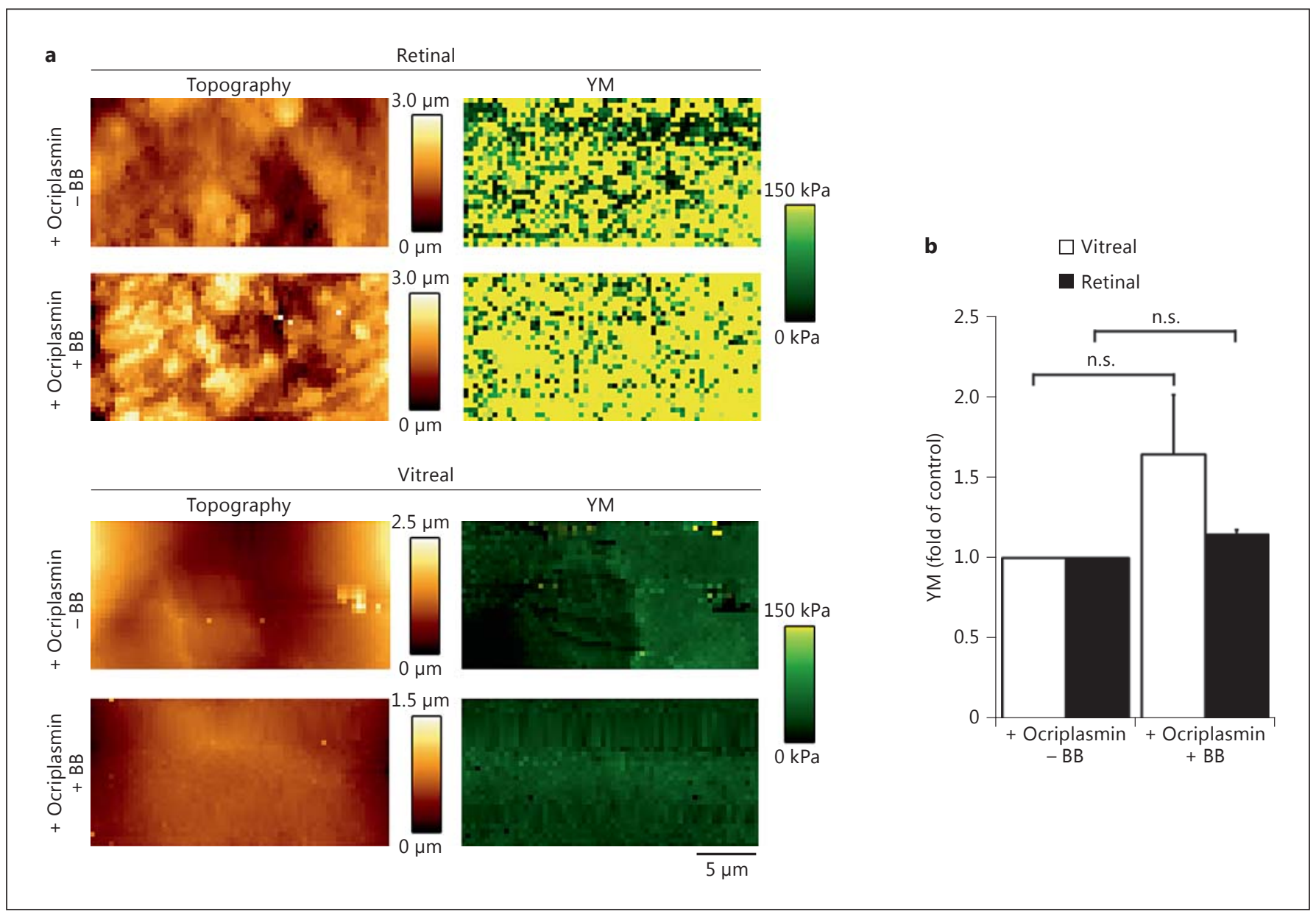

Fig. 3. a Mapas de topografía y elasticidad de 3 pacientes tratados con ocriplasmina antes y después de recibir de forma adicional AB. b Promedio de los mapas del MY de ambos lados de la MLI. El MY antes de la administración de $\mathrm{AB}$ se estableció a 1 y se comparó con el valor tras la administración de $\mathrm{AB}$. El AB no alteró significativamente el MY en los pacientes tratados con ocriplasmina.

fología de la MLI no se ve alterada por la administración de AB. Además, se manifestó un ligero aumento, aunque no significativo, de la elasticidad al comparar ambas partes antes y después de la administración de $A B$ en estos ojos (fig. 3b).

\section{Discusión}

Dado que la laminina constituye una familia de glucoproteínas que forman parte del andamiaje estructural de las membranas basales [15-18] y la ocriplasmina ejerce una actividad proteolítica en la laminina, el propósito de este estudio comprendía investigar el efecto enzimático de la ocriplasmina en las propiedades biomecánicas de la
MLI. Incluimos muestras de MLI extraídas de ojos sometidos a vitrectomía con disección de la MLI con y sin tinción de la MLI utilizando ABG tras recibir sin éxito tratamiento con ocriplasmina y las comparamos con muestras extraídas de ojos que no habían recibido inyección de ocriplasmina antes de someterse a cirugía.

El presente estudio coincide y confirma el resultado de nuestro informe previo con mediciones del microscopio de fuerza atómica tras la tinción de la MLI con tintes vitales [10]. De acuerdo con nuestra investigación anterior, confirmamos un efecto de rigidez de la MLI tras la administración de $A B$, que no se observaba en muestras de MLI no teñidas. Además, observamos un efecto de rigidez de la MLI más pronunciado tras el uso de indocianina verde en comparación con $\mathrm{ABG}$, especialmente tras la 
iluminación de la muestra teñida. Esto podría estar relacionado con el hecho de que la indocianina verde posee propiedades fotosintetizadoras que podrían aumentar este efecto. Además, observamos diferencias en la elasticidad tisular a la hora de analizar la superficie de la retina y el vítreo de la MLI tanto en muestras teñidas como no teñidas, y se obtuvo como resultado que la superficie retiniana de la MLI era más rígida que la vítrea. La confirmación de nuestros resultados anteriores garantiza que la microscopia de fuerza atómica representa una técnica fiable con resultados reproducibles. Partiendo de nuestros resultados, confiamos en que el microscopio de fuerza atómica habría detectado un posible efecto enzimático de la ocriplasmina en la MLI que interfiere con la rigidez del tejido, en caso de que estuviera estado presente.

Actualmente, la ocriplasmina se utiliza y está aprobada para el tratamiento de agujeros maculares pequeños y TVM inferior a $1.500 \mu \mathrm{m}$ mediante inyección intravítrea [2]. La enzima, administrada de manera intravítrea, ha demostrado inducir un desprendimiento posterior y licuación del vítreo. Algunos informes clínicos y pequeñas series de casos manifestaron inquietud acerca de los posibles efectos adversos de la enzima. Estos estudios publicaron información sobre síntomas y resultados clínicos que incluyeron distintos grados de disfunción retiniana, tales como pérdida de agudeza visual, dicromatopsia, nictalopía, estrechamiento del campo visual, anomalías o defectos externos de la señal retiniana en tomografía de coherencia óptica de dominio espectral, crecimiento del agujero macular, desprendimiento macular con acumulación del fluido subretiniano y cambios electrorretinográficos [6, 7, 12-14, 19-25].

La MLI de la retina es una estructura multilaminar compuesta principalmente de colágeno tipo IV, laminina, fibronectina y representa en esencia la membrana basal de células de Müller. Con un mayor aumento, suele revelar una superficie vítrea lisa y una superficie rugosa que se orienta hacia la retina. Esta apariencia morfológica diferente ayuda a identificar la superficie respectiva durante los estudios por microscopia como los que aquí se describen. Aunque de forma experimental se demostró que la ocriplasmina parece no afectar al aspecto estructural de la superficie de la parte vítrea de la MLI mediante microscopia electrónica [1], no contamos con información sobre las posibles alteraciones de las propiedades biomecánicas de la MLI tras el contacto de la superficie de la retina con la ocriplasmina, lo que puede ser perfectamente no detectable con el microscopio. Dichas alteraciones pueden ser consecuencia de las propiedades enzimáticas que se conocen de la ocriplasmina, siendo la la-

Propriedades de la MLI tras recibir tratamiento intravítreo con ocriplasmina minina y la fibronectina importantes dianas enzimáticas. Tanto la laminina como la fibronectina también constituyen componentes importantes de la MLI. La proteólisis de estas estructuras puede que permita incluso que el fármaco penetre en la MLI y alcance las capas externas de la retina neurosensorial, escindiendo la laminina a lo largo de las capas retinianas [12-14]. Destacamos además que no solo se indicó que la laminina constituye un componente de la MLI, tal y como se mencionó, sino también de la capa plexiforme externa, la membrana limitante externa y la matriz interfotorreceptora [26-28]. Aunque la división de más de una proteína puede ser la causa de varias manifestaciones de disfunción retiniana por ocriplasmina, se planteó la hipótesis de que la degradación de la laminina intrarretiniana desempeña una importante función en los efectos secundarios asociados a la ocriplasmina [12-14].

No obstante, la presente investigación posee ciertas limitaciones. Cabe destacar que el microscopio de fuerza atómica proporciona mediciones de la elasticidad de la superficie tisular, que tan solo informa de manera indirecta sobre los aspectos más profundos de la MLI. Sin embargo, no observamos ningún cambio en la rigidez de la MLI tras la exposición a ocriplasmina y parece bastante razonable asumir que es poco probable que suceda una afección de capas más profundas de la MLI a raíz de este resultado. Aún más importante, las mediciones de la microscopia de fuerza atómica, tal y como se ha realizado en el presente estudio, no representan las mediciones directas de los cambios de fuerza de tracción de la MLI. No obstante, puesto que la MLI representa una malla estructurada de forma irregular de varias fibras, se podrían transferir los resultados y concluir que resulta muy improbable que ocurra un cambio de las propiedades de tracción.

\section{Agradecimientos}

Queremos agradecer a JPK el apoyo técnico y constructivo proporcionado y a Andrea Wehmeyer su excelente asistencia técnica brindada.

\section{Declaración de situación}

Los autores indican que no existen conflictos de interés financiero.
Ophthalmologica 2017;238(suppl 1):1-8 DOI: $10.1159 / 000477510$ 


\section{Referencias bibliográficas}

1 Gandorfer A, Rohleder M, Sethi C, et al: Posterior vitreous detachment induced by microplasmin. Invest Ophthalmol Vis Sci 2004;45: 641-647.

2 Stalmans P, Benz MS, Gandorfer A, et al; MIVI-TRUST Study Group: Enzymatic vitreolysis with ocriplasmin for vitreomacular traction and macular holes. N Engl J Med 2012; 367:606-615.

3 Bartz-Schmidt K-U, Bertram B, Bornfeld N, et al: Current Statement of the German Ophthalmological Society, the Retina Society and the Professional Association of German Ophthalmologists for therapeutic intravitreal application of Ocriplasmin (JETREA ${ }^{\circledR}$ ) in ophthalmology (May 2013) (in German). Klin Monbl Augenheilkd 2013;230:629-634.

4 Stalmans P, Duker JS, Kaiser PK, et al: OCTbased interpretation of the vitreomacular interface and indications for pharmacologic vitreolysis. Retina 2013;33:2003-2011.

5 Haller JA, Stalmans P, Benz MS, et al: Efficacy of intravitreal ocriplasmin for treatment of vitreomacular adhesion. Subgroup analyses from two randomized trials. Ophthalmology 2015;122:117-122.

6 Kaiser PK, Kampik A, Kuppermann BD, et al: Safety profile of ocriplasmin for pharmacologic treatment of symptomatic vitreomacular adhesion/traction. Retina 2015;35:11111127.

7 Maier M, Abraham S, Frank C, Feucht N, Lohmann CP: Ocriplasmin as a treatment option for symptomatic vitreomacular traction with and without macular hole. First clinical experiences (in German). Ophthalmologe 2015;112:990-994.

8 Halfter W, Oertle P, Monnier CA, et al: New concepts in basement membrane biology. FEBS J 2015;282:4466-4479.
9 Halfter W, Candiello J, Hu H, et al: Protein composition and biomechanical properties of in vivo-derived basement membranes. Cell Adh Migr 2013;7:64-71.

10 Haritoglou C, Mauell S, Benoit M, et al: Vital dyes increase the rigidity of the internal limiting membrane. Eye 2013;27:1308-1315.

11 Henrich PB, Monnier CA, Halfter W, et al: Nanoscale topographic and biomechanical studies of the human internal limiting membrane. Invest Ophthalmol Vis Sci 2012;53: 2561-2570.

12 Marshall GE, Konstas AG, Bechrakis NE, Lee WR: An immunelectron microscope study of the aged human lens capsule. Exp Eye Res 1992;54:393-401.

13 Slattery AD, Blanch AJ, Quinton JS, Gibson CT: Accurate measurement of Atomic Force Microscope cantilever deflection excluding tip-surface contact with application to force calibration. Ultramicroscopy 2013;131:4655 .

14 Bilodeau GG: Regular pyramid punch problem. J App Mech 1992;59:519-523.

15 Hertz H: Über die Berührung fester elastischer Körper. Journal für reine und angewandte Mathematik 1881;92:156-171.

16 Hahn P, Chung MM, Flynn HW, et al: Safety profile of ocriplasmin for symptomatic vitreomacular adhesion. A comprehensive analysis of premarketing and postmarketing experiences. Retina 2015;35:1128-1134.

17 Tibbetts MD, Reichel E, Witkin AJ: Vision loss after intravitreal ocriplasmin: correlation of spectral-domain optical coherence tomography and electroretinography. JAMA Ophthalmol 2014;132:487-490.

18 Hager A, Seibel I, Riechardt A, Rehak M, Joussen AM: Does ocriplasmin affect the RPE-photoreceptor adhesion in macular holes? Br J Ophthalmol 2015;99:635-638.
19 Casswell E, Fernandez-sanz G, Mitry D, Luk S, Zakir R: Macular hole progression following ocriplasmin intravitreal injection. Case Rep Ophthalmol Med 2014;2014:403461.

20 Johnson MW: Acute ocriplasmin retinopathy. Retina 2015;35:1055-1058.

21 Fahim AT, Khan NW, Johnson MW: Acute panretinal structural and functional abnormalities after intravitreal ocriplasmin injection. JAMA Ophthalmol 2014;132:484-486.

22 Beebe DC: Understanding the adverse effects of ocriplasmin. JAMA Ophthalmol 2015;133: 229-231.

23 Singh RP, Li A, Debi R, et al: Anatomical and visual outcomes following ocriplasmin treatment for symptomatic vitreomacular traction syndrome. Br J Ophthalmol 2014;98:356-360.

24 Itoh Y, Kaiser PK, Singh RP, Srivastava SK, Ehlers JP: Assessment of retinal alterations after intravitreal ocriplasmin with spectral domain-optical coherence tomography. Ophthalmology 2014;121:2506-2507.e2.

25 Warrow DJ, Lai MM, Patel A, et al: Treatment outcomes and spectral-domain optical coherence tomography findings of eyes with symptomatic vitreomacular adhesion treated with intravitreal ocriplasmin. Am J Ophthalmol 2015;159:20-30.

26 Chen W, Mo W, Sun K, et al: Microplasmin degrades fibronectin and laminin at the vitreoretinal interface and outer retina during enzymatic vitrectomy. Curr Eye Res 2009;34: 1057-1064.

27 Libby RT, Champliaud MF, Claudepierre T, et al: Laminin expression in adult and developing retinae: evidence of two novel CNS laminins. J Neurosci 2000;20:6517-6528.

28 Libby RT, Lavallee CR, Balkema GW, et al: Disruption of laminin beta2 chain production causes alterations in morphology and function in the CNS. J Neurosci 1999;19:9399-9411. 